\title{
Multi-focus Image Fusion Using an Effective Discrete Wavelet Transform Based Algorithm
}

\author{
Yong Yang ${ }^{1}$, Shuying Huang ${ }^{2}$, Junfeng $\mathrm{Gao}^{3}$, Zhongsheng Qian ${ }^{1}$ \\ ${ }^{1}$ School of Information Technology, Jiangxi University of Finance and Economics, Nanchang 330013, China, \\ greatyangy@126.com; changesme@163.com \\ ${ }^{2}$ School of Software and Communication Engineering, Jiangxi University of Finance and Economics, Nanchang 330013, \\ China, shuyinghuang2010@126.com, (corresponding author) \\ ${ }^{3}$ College of Biomedical Engineering, South-Central University for Nationalities, Wuhan 430074, China, \\ junfengmst@163.com
}

In this paper, by considering the main objective of multi-focus image fusion and the physical meaning of wavelet coefficients, a discrete wavelet transform (DWT) based fusion technique with a novel coefficients selection algorithm is presented. After the source images are decomposed by DWT, two different window-based fusion rules are separately employed to combine the low frequency and high frequency coefficients. In the method, the coefficients in the low frequency domain with maximum sharpness focus measure are selected as coefficients of the fused image, and a maximum neighboring energy based fusion scheme is proposed to select high frequency sub-bands coefficients. In order to guarantee the homogeneity of the resultant fused image, a consistency verification procedure is applied to the combined coefficients. The performance assessment of the proposed method was conducted in both synthetic and real multi-focus images. Experimental results demonstrate that the proposed method can achieve better visual quality and objective evaluation indexes than several existing fusion methods, thus being an effective multi-focus image fusion method.

Keywords: Multi-focus image fusion, wavelet coefficients, image sharpness, energy.

\section{INTRODUCTION}

$\mathrm{O}$ VER THE past decade, with the rapid development of sensor technology, it is possible to have several images of the same scene providing complementary and redundant information although the scene is the same. This is because each image has been captured with a different sensor. Due to the limited depth of field of optical lenses in CCD devices, it is often impossible to get an image that contains all relevant objects in focus, which means if one object in the scene is in focus, another one will be out of focus (blurred) [1]. The popular way to solve this problem is multi-focus image fusion, which integrates multiple images of different focusing goal at the same scene into a composite focusing sharp image so that the new image is more suitable for visualization, detection or recognition tasks [2].

Up to now, many multi-focus image fusion methods have been developed. The methods can be generally classified into spatial domain and transform domain based techniques [3]. Several detailed surveys on this issue can be seen from references [4]-[6]. The simplest fusion method is to take the average of the two images pixel by pixel. However, this method usually leads to undesirable side effect such as reduced contrast [7]. Artificial neural network (ANN) has been introduced to realize multi-focus image fusion, as seen in reference [8]. However, the performance of ANN depends on the sample images and this is not an appealing characteristic. Later, Li et al. [9] proposed another regionbased multi-focus image fusion method. In their method, the source images are segmented at first, then the obtained regions are fused using their spatial frequency properties. However, this kind of method is usually complicated and time consuming, which are of vital importance to the fusion quality. Zhang et al. [10] proposed a multi-focus image fusion scheme based on blurring measure, which is used to locate the regions based on the blocking degree. The disadvantage of this block-based fusion method is that it suffers from blockness in the fused image. More recently, Pulse coupled neural network (PCNN) has also been introduced to implement image fusion, as seen in reference [11]. However, the PCNN technique as the region-based fusion method is also complicated and time-consuming. Due to the multiresolution transform can contribute a good mathematical model of human visual system (HVS) and can provide information on the contrast changes, the multiresolution techniques have then attracted more and more interest in image fusion. Meanwhile, the multiresolution based fusion method can produce good result in less computation time using less memory [12].

The multiresolution techniques involve two kinds, one is pyramid transform another is wavelet transform. Examples of pyramid approach include the Laplacian pyramid, the contrast pyramid, the gradient pyramid, etc [13]. However, for the reason of the pyramid method fails to introduce any spatial orientation selectivity in the decomposition process, the above pyramid based methods often cause blocking effects in the fusion results [7]. Another family of the multiresolution fusion techniques is the wavelet based method, which usually used the discrete wavelet transform (DWT) in the fusion. Since the DWT of image signals produces a nonredundant image representation, it can provide better spatial and spectral localization of image information as compared to other multiresolution representations [14]. Therefore, the DWT based method has been popular widely used for remote sensing image fusion, medical image fusion, as well as for multi-focus image fusion [15]-[20]. 
In the DWT based image fusion, the input images are first transformed into their multiresolution representations. The fusion process then creates a new composite multiresolution representation from these inputs by a certain fusion rule. The fused image is finally reconstructed by performing an inverse DWT (IDWT). Therefore, we can find the key step for DWT based image fusion is the definition of the fusion rule. The widely used fusion rule is the maximum selection (MS) scheme [7]. This simple scheme just selects the largest absolute wavelet coefficient at each location from the input images as the coefficient at the location in the fused image. However, as we know that the noise and artifacts usually has higher salient features in the image, therefore, this method is sensitive to noise and artifacts. For multi-focus image fusion, Chen et al. [20] proposed another fusion rule that selected the coefficients in the low frequency band by a weighted average scheme, and selected the coefficients in the high frequency bands by the MS scheme. The main drawback of such a method is that it will lead to the Gibbs phenomenon. Besides, the conventional fusion algorithms of MS, and Chen et al. [20] are a mainly pixel-based approach which means each individual decomposed coefficient is treated independently. However, as we know that even a very small error in registration will lead to mismatch of all the pixels in consideration. Therefore, all these methods are not robust against misregistration. To overcome the limitation of pixel-based algorithm, Chu et al. [17] proposed a window-based algorithm for multi-focus image fusion. However, in their method, they only fused the coefficients in low frequency sub-bands by using the local gradient, while the coefficients in high frequency sub-bands are still fused by the pixel-based MS algorithm.

In this paper, motivated by the fact that the key challenge of multi-focus image fusion is how to evaluate the blur of the image and then select information from the sharp image [21], and considering the physical meaning of the wavelet coefficients, a novel DWT based multi-focus image fusion method is presented. The main contribution of this work is that after executing DWT, the coefficients in low frequency sub-bands and high frequency sub-bands are treated by using different window-based fusion rules followed by a consistency verification process. The performance superiority of the proposed fusion approach is verified, when compared to that of several existing fusion methods, via experimental results using both subjective and objective fusion evaluations.

The rest of this paper is organized as follows. Section 2 proposes the new DWT based multi-focus image fusion algorithm in detail. Experimental results and discussion are given in Section 3 and the concluding remarks are presented in Section 4.

\section{THE PROPOSED FUSION METHOD}

As point out previously, the key point of multi-focus image fusion is to decide which portions of each image are in better focus than their respective counterparts in the associated images and then combine these regions to construct a well-focused image by certain fusion rules, which play an important role in DWT based fusion method. The window-based fusion rules usually consider the neighboring pixels in making decision choice, so they are superior to conventional pixel-based fusion rules. In addition, since the coefficients in low frequency and high frequency bands have different physical meanings, they should be treated by the combination algorithm through different procedures. Based on the above analysis, the procedure of our new DWT based multi-focus image fusion method is then presented, which is summarized in Table 1.

Table 1. The proposed Multi-focus image fusion algorithm.

Procedure:

Input: the aligned source images to be fused.

Step 1) Decompose the images by DWT into low frequency sub-bands and high frequency sub-bands.

Step 2) Perform different fusion rules to select the coefficients in low frequency sub-bands and high frequency sub-bands: the former are fused by a maximum sharpness based scheme, and the latter are fused by a maximum energy based scheme.

Step 3) All the coefficients are performed by a windowbased consistency verification process.

Step 4) The IDWT is applied to the final fused coefficients. Output: the fused image.

The basic idea of our method is to perform DWT on each source image in the first step; then after taking into account the characteristics of multi-focus images and their decomposition sub-bands, this paper presents a new fusion rule that treats the coefficients of the low frequency and high frequency sub-bands separately by using the fusion schemes as: the former are performed by a maximum sharpness based strategy, while the latter is performed by a maximum energy based strategy. In order to overcome the presence of noise and guarantee the homogeneity of the fused image, all the coefficients are subsequently performed by a window-based consistency verification process. Finally, the fused image is obtained by performing the IDWT on the combined wavelet coefficients. It should be noted that the high frequency sub-bands include the vertical, horizontal, and diagonal high frequencies of the image, respectively. Therefore, the fusion process must be performed in all these domains.

To simplify the description of the different alternatives available in forming a fusion rule, as reference [16] we also consider only two source images, $X$ and $Y$, and the fused image $Z$. The method can of course be easily extended to more than two images. Generally, an image $I$ has its multiscale decomposition (MSD) representation denoted as $\mathrm{D}_{I}$. Hence we will encounter $\mathrm{D}_{X}, \mathrm{D}_{Y}$, and $\mathrm{D}_{Z}$. Let $p=(m, n$, $k, l)$ indicate the index corresponding to a particular MSD coefficient, where $m$ and $n$ indicate the spatial position in a given frequency band, $k$ is the decomposition level, and $l$ is the frequency band of the MSD representation. Therefore, $\mathrm{D}_{I}(p)$ denotes the MSD value of the corresponding coefficient. 


\section{A. Fusion for low frequency sub-band coefficients}

The low frequency sub-band is the original image at the coarser resolution level, which can be considered as a smoothed and subsampled version of the original image. Most information of their source images is kept in the low frequency sub-band. As we know that for multi-focus images, each of them has some clear information about the same scene but none of them is sufficient in terms of its information contents. The main idea of their fusion is to select sharply pixels from source images and combine them together to reconstruct the superior image in which all the pixels can be clearly focused. Therefore, here we proposed to employ a sharpness focus measure to select the coefficients in low frequency sub-bands. The sharpness measure is a Tenengrad function based on Sobel operator, which also considered the neighborhood information of a pixel by a fixed window. This measure can be used to indicate the clarity of the image pixels, and a well-focused image is expected to have sharper information. The sharpness focus measure is defined as:

$$
\nabla G(p)=\left[\nabla G_{m}(p)^{2}+\nabla G_{n}(p)^{2}\right]^{1 / 2}
$$

where $\nabla G_{m}(p), \nabla G_{n}(p)$ can be express by

$$
\begin{aligned}
& \nabla G_{m}(p)=\left\{\begin{array}{l}
-D(m-1, n-1, k, l)-2 D(m-1, n, k, l) \\
-D(m-1, n+1, k, l)+D(m+1, n-1, k, l) \\
+2 D(m+1, n, k, l)+D(m+1, n+1, k, l)
\end{array}\right\} \\
& \nabla G_{n}(p)=\left\{\begin{array}{l}
D(m-1, n-1, k, l)+2 D(m, n-1, k, l) \\
+D(m+1, n-1, k, l)-D(m-1, n+1, k, l) \\
-2 D(m, n+1, k, l)-D(m+1, n+1, k, l)
\end{array}\right\}
\end{aligned}
$$

After obtaining the sharpness of all pixels of the low frequency sub-bands, a maximum scheme of it is then performed. Because, for multi-focus image fusion, the focused pixels should produce maximum sharpness measure, yet the defocused pixels should produce minimum sharpness measure on the contrary. The fusion scheme of the coefficients in low frequency sub-band is then defined as:

$$
D_{Z}(p)= \begin{cases}D_{X}(p) & \nabla G_{X}(p) \geq \nabla G_{Y}(p) \\ D_{Y}(p) & \nabla G_{X}(p)<\nabla G_{Y}(p)\end{cases}
$$

\section{B. Fusion for high frequencys sub-band coefficients.}

The high frequency sub-bands contain the detail coefficients of an image, which usually have large absolute values correspond to sharp intensity changes and preserve salient information in the image. So, if the rule mentioned above for low frequency sub-bands is adopted here, the fused results will be blocked. Besides, according to the wavelet transform theory, we know that the energy of the high frequency coefficients of a clear image is much larger than that of a blurred one. Based on this analysis and considering that the wavelet coefficient is related to its neighboring region, we propose a fusion scheme by computing the neighboring energy maximum to select the high frequency coefficients. The neighboring energy feature is defined as:

$$
N E(p)=\sum_{(i, j) \in W} H(i, j) D^{2}(m+i, n+j, k, l)
$$

where $W$ is the $3 \times 3$ neighboring size, and $H(i, j)$ denotes the weighted template, which is used to highlight the center pixel of the window and is set as:

$$
H(i, j)=\left[\begin{array}{ccc}
0 & 1 / 8 & 0 \\
1 / 8 & 1 / 2 & 1 / 8 \\
0 & 1 / 8 & 0
\end{array}\right]
$$

Then we perform a maximum selection rule on the coefficients in high frequency sub-bands, which select the coefficients with higher energy into the fused image. The fusion scheme used for it is expressed as:

$$
D_{Z}(p)= \begin{cases}D_{X}(p) & N E_{X}(p) \geq N E_{Y}(p) \\ D_{Y}(p) & N E_{X}(p)<N E_{Y}(p)\end{cases}
$$

\section{Consistency verification}

As can be seen from above subsections all the coefficients of both low frequency and high frequency bands are selected by the maximum selection schemes, but as we know that the maximum selection technique will be influent in case of noise. Furthermore, since we cope with the coefficients separately, this method cannot guarantee the homogeneity in the resultant fused image. Therefore, a consistency verification scheme is then performed, which can also ensure the dominant features are incorporated as completely as possible into the fused image [7]. The idea of this attempt is likely to be a majority filter. In this paper, we apply a window-based verification (WBV) to the coefficients in the composite MSD. The WBV employs a small window centered at the current coefficient position. The WBV rule is that if the center composite MSD coefficient comes from image $\mathrm{X}$, but the majority of the surrounding coefficients in the window come from image $\mathrm{Y}$, then the center sample is changed to come from $\mathrm{Y}$, and vice versa. In the implementation, this rule is applied to a binary decision map, and then it is followed by the application of a majority filter. The fused coefficients are finally obtained by the new binary decision map. This process can be formulated as follows.

$$
\begin{gathered}
D_{X}^{\prime}(p)=\max _{w \in W}\left(\mid D_{X}(p, w)\right), \\
D_{Y}^{\prime}(p)=\max _{w \in W}\left(D_{Y}(p, w)\right) . \\
q_{X}(p)=\left\{\begin{array}{cc}
1, & D_{X}^{\prime}(p)>D_{Y}^{\prime}(p) \\
0, & \text { otherwise }
\end{array}\right.
\end{gathered}
$$




$$
\begin{gathered}
q_{Y}(p)=\left\{\begin{array}{cc}
1, & D_{Y}^{\prime}(p) \geq D_{X}^{\prime}(p) \\
0, & \text { otherwise }
\end{array}\right. \\
q_{X}^{*}(p)=\left\{\begin{array}{cc}
1, & \sum q_{X}(p) \geq 5 \\
0, & \text { otherwise }
\end{array}\right. \\
q_{Y}^{*}(p)=1-q_{X}^{*}(p) \\
D_{Z}(p)=q_{X}^{*}(p) D_{X}(p)+q_{Y}^{*}(p) D_{Y}(p)
\end{gathered}
$$

Once all the coefficients are achieved from the above three procedures, the IDWT is then performed on them, the fused image is thus constructed.

\section{EXPERIMENTS AND ANALYSIS}

In this section, several fusion experiments were carried out on both synthetic and real multi-focus images. We have also compared our results with the conventional Filter-subtractdecimate (FSD) pyramid fusion method [22], the DWT based method proposed by Chen et al. [20], which is abbreviated as DWT method, and a bilateral sharpness criterion based method of [21]. As most references, in all test cases we assume the source images to be in perfect registration. A good survey of image registration can be seen in reference [23]. In the experiments, we use the Daubechies' db8 as the wavelet basis for DWT based method and the proposed method and their decomposition level is 3 .

\section{A. Synthetic date experiments}

To verify the effectiveness of the proposed method, the first experiment was conducted on two synthetic out-offocus images, which are created by convolving a Gaussian blurring with the popular widely used standard image Lena. Fig.1.(a) is the original image of size $256 \times 256$, which is served as the ideal reference image. Fig.1.(b) is the image blurred on the left, while Fig.1.(c) is the image blurred on the right. Figs.1.(d)-(h) are the fused results obtained by fusing Fig.1.(b) and Fig.1.(c) with the five methods mentioned above, respectively. From the five fused images it is easy to see that Figs.1.(d) and (e) have a lower contrast than Figs.1.(f) and (h), and Fig.1. (g) has some block effects at the edge of the image. However, it is difficult to discriminate the difference between the DWT method and the proposed method by visual inspection. Hence, a frequently used metric, root mean square error (RMSE) is employed here to objectively evaluate the performance of the methods. This metric can indicate how much error the fused image conveys about the reference image. Therefore, lower value of RMSE means better fused result. The RMSE is defined as:

$$
R M S E=\left(\frac{1}{M N} \sum_{n=1}^{N} \sum_{m=1}^{M}\left(x_{R}(n, m)-x_{F}(n, m)\right)^{2}\right)^{1 / 2}
$$

where $\mathrm{x}_{R}$ and $\mathrm{x}_{F}$ denote the ideal reference image and fused image, respectively, and $M$ and $N$ are the dimensions of the image. The RMSE values of the five different methods are calculated and shown in Fig.2. It can be seen from Fig.2. that the RMSE value of the proposed method is the smallest in the five methods, and the RMSE value of the FSD pyramid method is the largest. The quantitative results presented here can demonstrate that the proposed technique can fuse the image with conveying less error than other four methods.

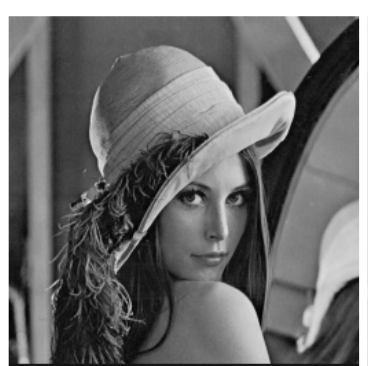

(a)

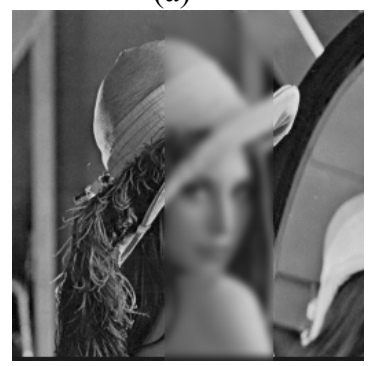

(c)

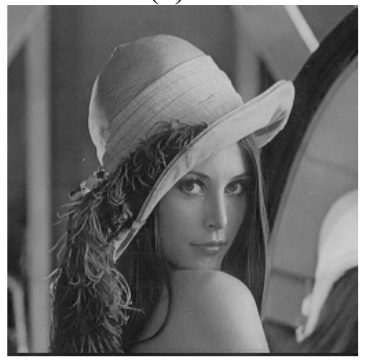

(e)

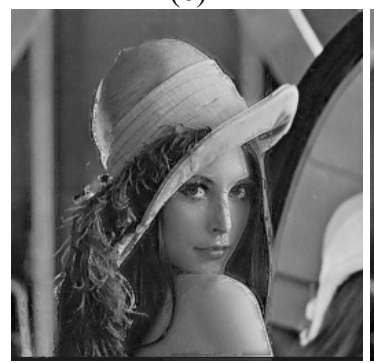

(g)

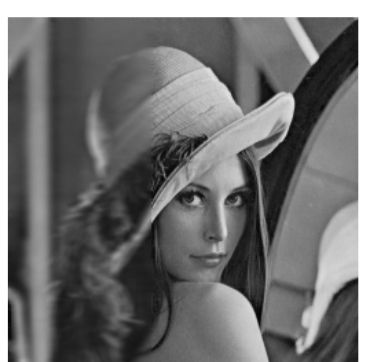

(b)

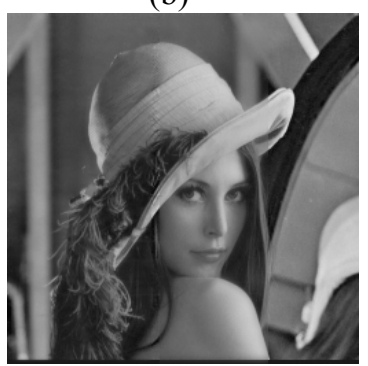

(d)

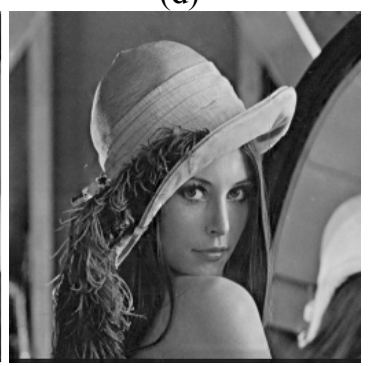

(f)

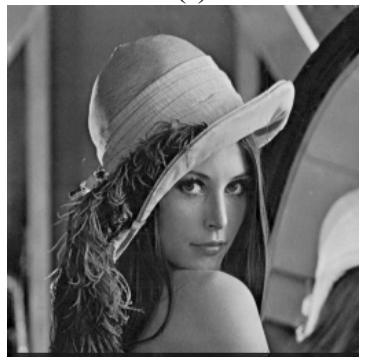

(h)
Fig.1. Image fusion with the simulated pair from Lena image. (a) The original image (reference image or ground truth); (b) image blurred on the left; (c) image blurred on the right; (d) fused image by pixel averaging; (e) fused image by FSD pyramid; (f) fused image by DWT; (g) fused image by the method of [21]; (h) fused image by the proposed method. 


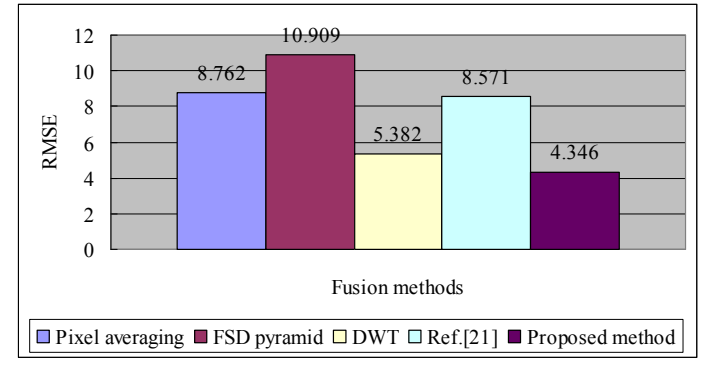

Fig.2. RMSE values of the five fusion methods in Fig.1.

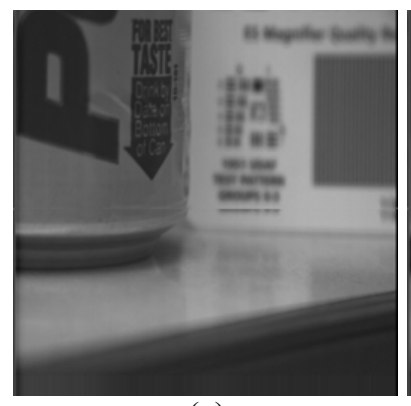

(a)

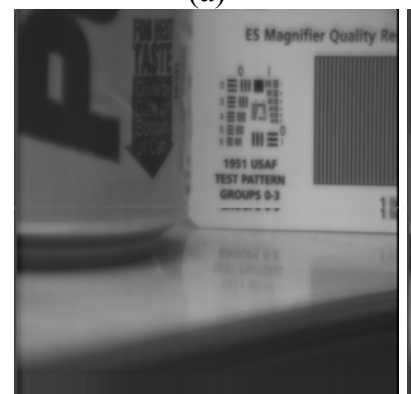

(c)

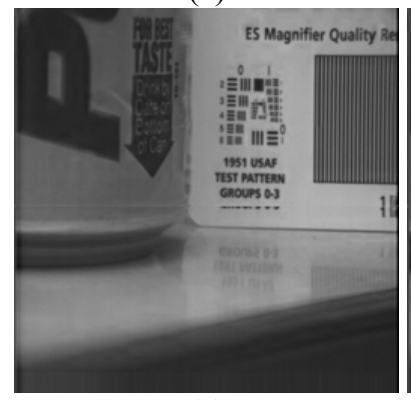

(e)

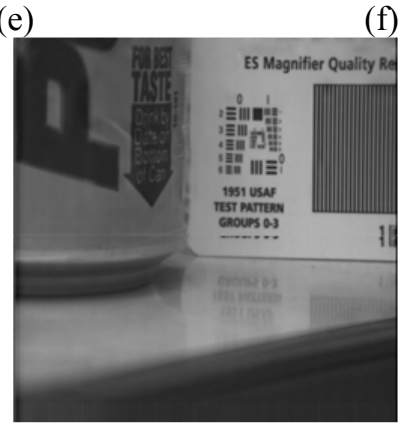

$(\mathrm{g})$

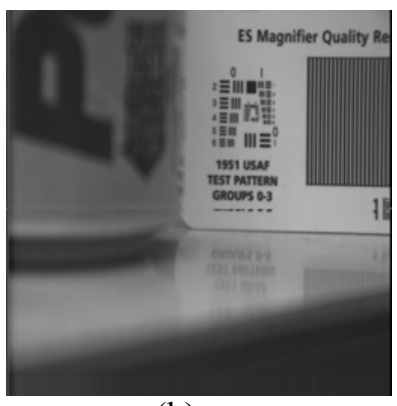

(b)

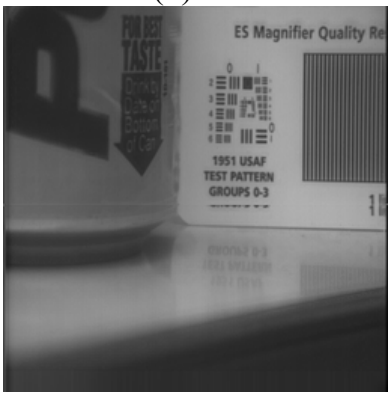

(d)

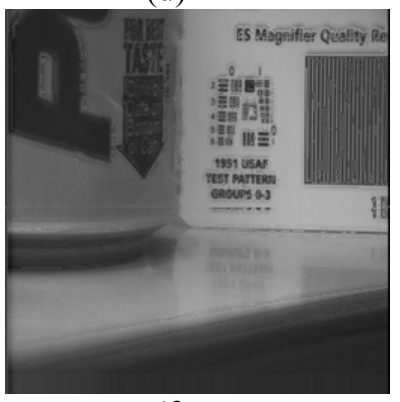

(f)
Fig.3. Fusion results of multi-focus Pepsi images with different methods. (a) Near focused image (focus on the Pepsi can); (b) far focused image (focus on the testing card); (c) fused image by pixel averaging; (d) fused image by FSD pyramid; (e) fused image by DWT; (f) fused image by the method of [21] ; (g) fused image by the proposed method.

\section{B. Real multi-focus data experiments}

In this section, two frequently used real multi-focus image samples were tested. The first group of images is a pair of Pepsi images, which contain multiple objects at difference distances from the camera as shown in Figs.3.(a) and (b). Fig.3.(a) is focused on the Pepsi can, while Fig.3.(b) is focused on the testing card. The two images are then fused by the above five methods and their resultant images are presented in Figs.3.(c)-(g), respectively. From the fusion results, we can easily observe that the results of the pixel averaging and FSD pyramid methods have a lower contrast than those of the DWT method, the method of [21], and the proposed method, for example, the texts in the testing card are not clear in Figs.3.(c) and (d), but they are clear in Figs.3.(e) and (g) except that Fig.3.(f) has some block effects. Again, it is hard to tell the difference between the results of the DWT method and the proposed method by subjective evaluation. Hence, in order to better evaluate these fusion methods, quantitative assessments of the performance of the five methods are needed. However, it should be noted that actually for image fusion it is often impossible to get the ideal or reference composite image, so the above RMSE metric cannot be used here. As a result, four other evaluation criteria including the stand variation (STD), the information entropy (IE), the $\mathrm{Q}^{\mathrm{AB} / \mathrm{F}}$, and the $\mathrm{Q}_{\mathrm{E}}$ metrics are then introduced and employed in the paper [2426].

The STD denotes the degree of deviation between the gray levels and its mean value for the overall image; the IE measures the richness of information in an image; the $\mathrm{Q}^{\mathrm{AB} / \mathrm{F}}$ metric reflects the quality of visual information obtained from the fusion of input images, and $\mathrm{Q}_{\mathrm{E}}$ metric is the edgedependent fusion quality index. Therefore, the larger their values, the better the performance.

The above four evaluation criteria are then applied to evaluate the five fusion methods in Fig.3., and the detailed quantitative results are given in Table 2. From Table 2., we can observe that the values of all quality indices of the proposed method are larger than those of pixel averaging method, FSD pyramid method, method of [21], and DWT method.

Table 2. Performance comparison of the five fusion methods in Fig.3.

\begin{tabular}{cccccc}
\hline & $\begin{array}{c}\text { Pixel } \\
\text { averaging }\end{array}$ & $\begin{array}{c}\text { FSD } \\
\text { pyramid }\end{array}$ & DWT & Ref.[21] & $\begin{array}{c}\text { Proposed } \\
\text { method }\end{array}$ \\
\hline STD & 43.638 & 42.769 & 44.278 & 44.068 & $\mathbf{4 4 . 4 9 5}$ \\
IE & 7.000 & 7.014 & 7.049 & 7.042 & $\mathbf{7 . 0 7 2}$ \\
$\mathrm{Q}^{\mathrm{AB} / \mathrm{F}}$ & 0.649 & 0.684 & 0.679 & 0.626 & $\mathbf{0 . 6 9 5}$ \\
$\mathrm{Q}_{\mathrm{E}}$ & 0.474 & 0.573 & 0.531 & 0.462 & $\mathbf{0 . 5 8 6}$ \\
\hline
\end{tabular}


Table 3. Performance comparison of the five fusion methods in Fig.4.

\begin{tabular}{cccccc}
\hline & $\begin{array}{c}\text { Pixel } \\
\text { averaging }\end{array}$ & $\begin{array}{c}\text { FSD } \\
\text { pyramid }\end{array}$ & DWT & Ref.[21] & $\begin{array}{c}\text { Proposed } \\
\text { method }\end{array}$ \\
\hline STD & 50.699 & 47.408 & 51.811 & 51.326 & $\mathbf{5 2 . 1 5 6}$ \\
IE & 7.276 & 7.369 & 7.392 & 6.962 & $\mathbf{7 . 4 0 2}$ \\
$\mathrm{Q}^{\mathrm{ABF}}$ & 0.668 & 0.675 & 0.674 & 0.594 & $\mathbf{0 . 6 8 6}$ \\
$\mathrm{Q}_{\mathrm{E}}$ & 0.460 & 0.470 & 0.460 & 0.409 & $\mathbf{0 . 4 9 0}$ \\
\hline
\end{tabular}

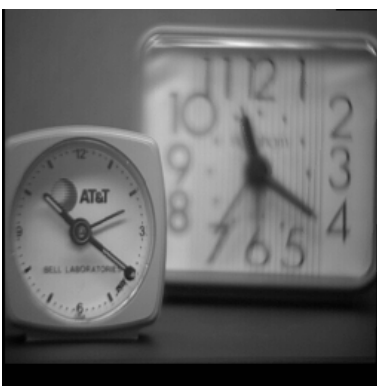

(a)

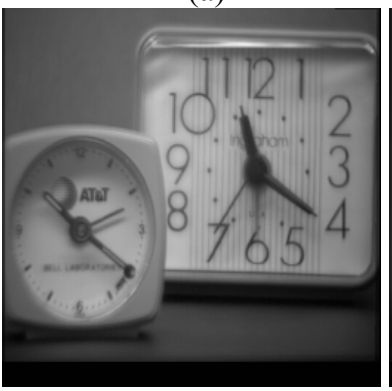

(c)
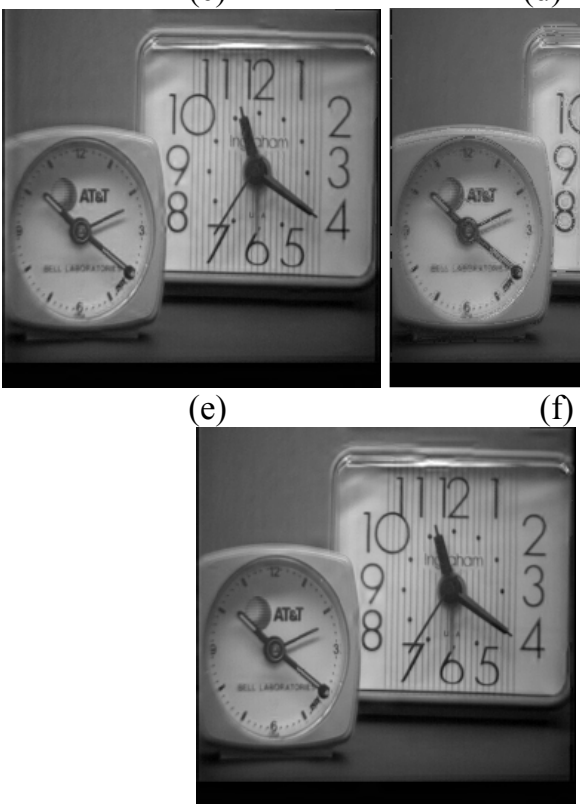

(g)

Fig.4. Fusion results of multi-focus Clock images with different methods. (a) Near focused image; (b) far focused image; (c) fused image by pixel averaging; (d) fused image by FSD pyramid; (e) fused image by DWT; (f) fused image by the method of [21]; (g) fused image by the proposed method.
The last examples are another pair of Clock images as shown in Figs.4.(a) and (b), which are obtained with two different focal planes. The fusion experiments are performed by using the five methods, and their corresponding fused results are depicted in Figs.4.(c)-(g), respectively. By subjective evaluation, we can observe that the results of DWT and the proposed methods are better than those of pixel averaging, FSD pyramid, and [21] methods. Also, as the second experiment we carried out the quantitative assessments for the five methods with the four criteria mentioned above, and their evaluation results are given in Table 3. From Table 3., we can see that just as Table 2. the proposed method provides the best performance and outperforms the other four methods in terms of values of the STD, IE, $\mathrm{Q}^{\mathrm{AB} / \mathrm{F}}, \mathrm{Q}_{\mathrm{E}}$. Therefore, based on these two groups of real data experiments, we can conclude that all the quantitative evaluations are basically corresponding to the visual effects, and the proposed DWT based fusion method performs the best in the five fusion methods.

\section{CONCLUSION}

The aim of multi-focus image fusion is to fuse multiple images that are captured using different camera settings of the same scene to construct a superior image, in which all the objects are well focused and their information is complete. Viewing this and considering the different physical meaning of coefficients in approximation and detailed images, this paper presents a simple yet effective DWT based algorithm for multi-focus image fusion. The main contribution of this work is that we have set a novel fusion rule for selecting the coefficients in the DWT domain followed by a consistency verification process. In the method, the fusion scheme of low frequency coefficients is based on a maximum sharpness based algorithm, while for high frequency coefficients, a maximum energy based selection algorithm is employed.

A series of experiments on evaluating the fusion performance have been made and the results show that the proposed method outperforms several fusion methods, including the pixel averaging method, FSD pyramid method, an existing bilateral sharpness criterion based method, and a DWT based method, both in visual effect and objective evaluation criteria. In the future, we will extend the proposed algorithm to fuse other multi-sensor images, such as medical images, remote sensing images, and so on.

\section{ACKNOWLEDGEMENTS}

This work was supported by the National Natural Science Foundation of China (61262034, 81271659, 61262010), by the Key Project of Chinese Ministry of Education (211087), by the Natural Science Foundation of Jiangxi Province (20114BAB211020, 20132BAB201025), by the Young Scientist Foundation of Jiangxi Province (20122BCB23017), and by the Science and Technology Research Project of the Education Department of Jiangxi Province ( GJJ13302). 


\section{REFERENCES}

[1] Shah, P., Merchant, S.N., Desai, U.B. (2013). Multifocus and multispectral image fusion based on pixel significance using multiresolution decomposition. Signal Image and Video Processing, 7 (1), 95-109.

[2] Chai, Y., Li, H.F., Li, Z.F. (2011). Multifocus image fusion scheme using focused region detection and multiresolution. Optics Communications, 284 (19), 4376-4389.

[3] Zhang, B.H., Zhang, C.T., Liu, Y.Y., Wu, J.S., He, L. (2014). Multi-focus image fusion algorithm based on compound PCNN in Surfacelet domain. Optik, 125 (1), 296-300.

[4] Goshtasby, A.A., Nikolov, S.G. (2007). Image fusion: Advances in the state of the art. Information Fusion, 8 (2), 114-118.

[5] Smith, M.I., Heather, J.P. (2005). Review of image fusion technology in 2005. In Thermosense XXVII. SPIE, Vol. 5782, 29-45.

[6] Yang, B., Jing, Z.L., Zhao, H.T. (2010). Review of pixel-level image fusion. Journal of Shanghai Jiaotong University (Science), 15 (1), 6-12.

[7] Li, H., Manjunath, B.S., Mitra, S.K. (1995). Multisensor image fusion using the wavelet transform. Graphical Models and Image Processing, 57 (3), 235245.

[8] Li, S.T., Kwok, J.T., Wang, Y. (2002). Multifocus image fusion using artificial neural networks. Pattern Recognition Letters, 23 (8), 985-997.

[9] Li, S.T., Yang, B. (2008). Multifocus image fusion using region segmentation and spatial frequency. Image and Vision Computing, 26 (7), 971-979.

[10] Zhang, Y.J., Ge, L.L. (2009). Efficient fusion scheme for multi-focus images by using blurring measure. Digital Signal Processing, 19 (2), 186-193.

[11] Wang, Z.B., Ma, Y.D., Gu, J.S. (2010). Multi-focus image fusion using PCNN. Pattern Recognition, 43 (6), 2003-2016.

[12] De, I., Chanda, B. (2006). A simple and efficient algorithm for multifocus image fusion using morphological wavelets. Signal Processing, 86 (5), 924-936.

[13] Redondo, R., Sroubek, F., Fischer, S., Cristobal, G. (2009). Multifocus image fusion using the log-Gabor transform and a Multisize Windows technique. Information Fusion, 10 (2), 163-171.
[14] Petrovic, V.S., Xydeas, C.S. (2004). Gradient-based multiresolution image fusion. IEEE Transactions on Image Processing, 13 (2), 228-237.

[15] Liu, G.X., Yang, W.H. (2002). A waveletdecomposition-based image fusion scheme and its performance evaluation. Acta Automatica Sinica, 28 (6), 927-934.

[16] Pajares, G., Cruz, J.M.D.L. (2004). A wavelet-based image fusion tutorial. Pattern Recognition, 37 (9), 1855-1872.

[17] Chu, H., Li, J., Zhu, W.L. (2005). Multi-focus image fusion scheme with wavelet transform. OptoElectronic Engineering, 32 (8), 59-63.

[18] Zheng, Y.F., Essock, E.A., Hansen, B.C., Haun, A.M. (2007). A new metric based on extended spatial frequency and its application to DWT based fusion algorithms. Information Fusion, 8 (2), 177-192.

[19] Yang, Y., Park, D.S., Huang, S.Y., Yang, J.C. (2010). Fusion of CT and MR images using an improved wavelet based method. Journal of X-Ray Science and Technology, 18 (2), 157-170.

[20] Chen, Y.Q., Chen, L.Q., Gu, H.J., Wang, K. (2010). Technology for multi-focus image fusion based on wavelet transform. In Third International Workshop on Advanced Computational Intelligence, 25-27 August 2010. IEEE, 405-408.

[21] Tian, J., Chen, L., Ma, L.H., Yu, W.Y. (2011). Multifocus image fusion using a bilateral gradient-based sharpness criterion. Optics Communications, 284 (1), 80-87.

[22] Burt, P.J., Kolczynski, R.J. (1993). Enhanced image capture through fusion. In Fourth International Conference on Computer Vision, 1-14 May 1993. IEEE, 173-182.

[23] Deshmukh, M., Bhosle, U. (2011). A Survey of image registration. International Journal of Image Processing, 5 (3), 245-269.

[24] Haghighat, M.B.A., Aghagolzadeh, A., Seyedarabi, H. (2011). A non-reference image fusion metric based on mutual information of image features. Computers and Electrical Engineering, 37 (5), 744-756.

[25] Xydeas, C.S., Petrovic, V. (2000). Objective image fusion performance measure. Electronics Letters, 36 (4), 308-309.

[26] Piella, G., Heijmans, H. (2003). A new quality metric for image fusion. In International Conference on Image Processing (ICIP 2003), 14-17 September 2003. IEEE, 173-176.

Received July 30, 2013. Accepted March 24, 2014. 